\title{
Get Rapidly Increasing Reputation for the New Journal
}

\section{Shanyong Chen*}

Associate professor, Department of Mechatronic Engineering, National University of Defense Technology (NUDT), China

When celebrating the foundation of Advances in Robotics and Automation, we are also thinking over how to get rapidly increasing reputation for this new journal. The most frequently used measure of international reputation may be the journal impact factor (IF) or basically the citation frequency. However being suspicious of a new journal, authors would like to present their research to other journals of well established reputation. Therefore our top priority is to take measures to attract more research readers as well as distinguished authors with their original and innovative research.

Open access is a good solution. Readers are completely free to get and distribute the publications at any time, anywhere in the world, as long as they can access the internet. The power of internet is never overestimated. The more readers we have, the higher citation frequency we get, and then the higher reputation is established which in return attracts more distinguished authors. It is a beneficial cycle. Another benefit of open access is the quick publication, always presenting timely research state of the art to readers. As a good example, the open access journal Optics Express became a leading journal in optics just after several years of foundation, with IF even exceeding that of Optics Letters.
At the same time, we also note a mushroom growth of open access journals in different scientific fields. ARA must have some unique features to distinguish itself from others. On the one hand, we should make more advantages of OMICS Group special features such as companion multi-media, social networking and so forth. Sometimes it is an indispensible supplement for the publications and sometimes people really need a "face to face talk", even virtually. Nowadays pure literal communication between authors and readers is no longer sufficient.

On the other hand, publication fee is a big barrier especially at the start of a journal, in view of the fact that many reputed journals do not charge on publication. This barrier will not disappear until the journal has got a high reputation. My suggestion is to provide special discount to articles written on invitation and pay back to authors according to the download or citation frequency of their articles. It is co-win for both the journal and authors.

In a word, everything is difficult at the start, but we do not expect to make it at one stroke.
*Corresponding author: Shanyong Chen, Associate professor, Department of Mechatronic Engineering, National University of Defense Technology (NUDT), China, E-mail: shanyongchen@tom.com

Received January 24, 2012; Accepted February 06, 2012; Published February 08, 2012

Citation: Chen S (2012) Get Rapidly Increasing Reputation for the New Journal. - \$\$ GY5 RERM\$ XVRP DW:e101. doi:10.4172/2168-9695.1000e101

Copyright: @ 2012 Chen S. This is an open-access article distributed under the terms of the Creative Commons Attribution License, which permits unrestricted use, distribution, and reproduction in any medium, provided the original author and source are credited. 